\title{
Measuring up to industry
}

\author{
Nadya Anscombe talks to Wolfgang Osten, director of the Institute for Applied Optics, about the \\ challenges that optical metrology faces in keeping pace with demands from industry.
}

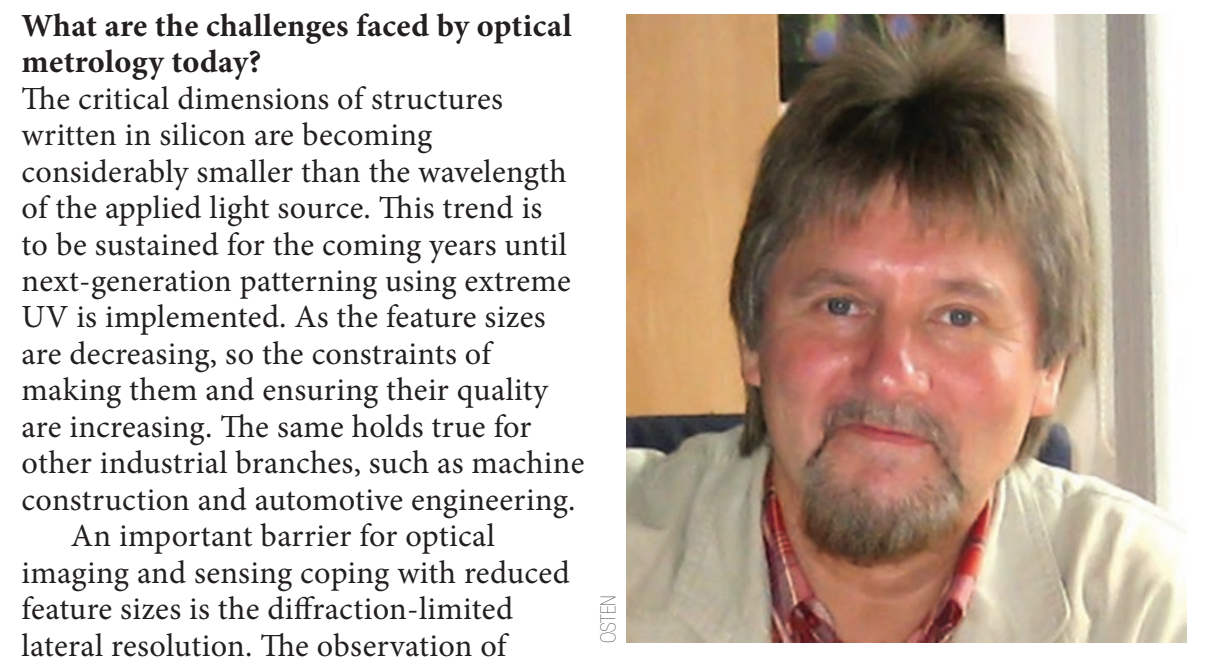

resolution. The observation of this physical limitation is of increasing importance, not only for microscopic techniques, but also for the application of three-dimensional measurement techniques on the wafer scale and at the macroscopic level. Other challenges include the reliable and precise measurement of free-form surfaces, both technical and optical, and the assurance of the traceability and the certified assessment of the uncertainty of the measurement results.

Tools to tackle these challenges include: new brilliant light sources with tunable properties; optoelectronic detectors with improved space-bandwidth product and spectral sensitivity; spatial light modulators with an ability for amplitude as well as phase control; and last but not least, the continuously growing computer power.

Optical metrology still remains the technique with the most potential, especially for miniature systems. It is a non-destructive, non-invasive and fast technique with many advantages over other technologies. However, some problems are still waiting for a solution. Objects showing high aspect ratio and non-cooperative surfaces (for example, shiny or translucent) are still a challenge.

What features are required? Today's manufacturing industry places huge demands on metrology systems. dimensions as well as other properties, such as line-edge roughness and layer thickness. However, both techniques are still struggling with complex threedimensional structures such as cross gratings. Scatterometry is particularly interesting, as its speed enables more than spot-checking, and in-line integration is on the way. However, the technique requires a great deal of computing power, which until now, was not readily available.

With dynamic miniature systems, such as microelectromechanical systems (MEMS) and microoptoelectromechanical systems, we need a metrology system that can test the system's response to an operational load. Digital holography can be used to investigate MEMS with a resolution in the nanometre range. Equipped with self-referencing techniques, such as shearing and special sensor arrays that can acquire several phase-shifted images simultaneously, the method can be made very robust against environmental influences. However, digital holography also depends strongly on the progress in sensor technology and computer power. Sensor chips with increased space-bandwidth product are badly needed to increase the range of objects that can be investigated.

What needs to happen to enable widespread adoption of this technology? This could be a long story. One current problem with optical metrology systems is that each one is unique and custommade to fit its application. Often a special environment needs to be implemented. Furthermore, in contrast to conventional tactile technologies, optical methods do not deliver a direct approach to the quantity of interest such as a profile height, which can make the system tricky and not user-friendly. We also need to make optical metrology systems with readings that are comparable to other technologies. In-line metrology and defectoscopy is a must for future production systems.

light. In addition to different types of microscopes, diffractometry and scatterometry are being introduced in the semiconductor industry for measuring
Nadya Anscombe is a freelance science and technology journalist based in the UK. 